\title{
Anxiety Reduction through Detachment: Subjective, Physiological, and Neural Effects
}

\author{
Raffael Kalisch, Katja Wiech, Hugo D. Critchley, \\ Ben Seymour, John P. O'Doherty, David A. Oakley, \\ Philip Allen, and Raymond J. Dolan
}

\begin{abstract}
The ability to volitionally regulate emotions helps to adapt behavior to changing environmental demands and can alleviate subjective distress. We show that a cognitive strategy of detachment attenuates subjective and physiological measures of anticipatory anxiety for pain and reduces
\end{abstract}

\section{INTRODUCTION}

Anxiety is a psychological, physiological, and behavioral response to anticipation of an aversive event (Gray \& McNaughton, 2000; Eysenck, 1997; Cannon, 1929). Anxiety also amplifies the psychological and physiological reaction to aversive events (Ploghaus, Narain, et al., 2001; Epstein \& Clarke, 1970). Thus, in addition to being subjectively unpleasant, anxiety has costs in that it competes for bodily and cognitive resources. A functional account of anxiety emphasizes its role in avoidance of anticipated aversive events (Hofer, 2002; Gray \& McNaughton, 2000). Due to its associated cost, anxiety may be maladaptive where an aversive event is unavoidable or where the anxiety response is exaggerated in relation to the harm an aversive event might cause. In such situations, the ability to volitionally regulate anxiety is advantageous.

Gross (2002) has provided a theoretical framework and nomenclature for research on emotion regulation. Building on a process model of emotion generation (Gross, 1999), he has identified putative emotion regulatory processes. Regulatory processes such as expressive suppression, which focus on modifying behavioral responses, are differentiated from processes bearing on the generation of emotional reactions (so-called antecedent-focused processes). Antecedent-focused strategies comprise selection and modification of an emotion-eliciting situation as well as cognitive strategies like focusing on particular aspects of a situation or reappraising its meaning (Gross, 2002).

University College London reactivity to receipt of pain itself. Using functional magnetic resonance imaging, we locate the potential site and source of this modulation of anticipatory anxiety in the medial prefrontal/anterior cingulate and anterolateral prefrontal cortex, respectively.

Gross (2002) has reviewed evidence that the response-focused strategy of expressive suppression is ineffective in attenuating experiential and physiological components of negative emotions and may even have adverse effects on subjective well-being and health. In the context of regulating maladaptive anxiety, antecedent-focused strategies, particularly cognitiveoriented strategies, are of special interest. In this article, we focus on reappraisal which has previously been shown to reduce both experiential and physiological aspects of anticipatory anxiety for pain (Holmes \& Houston, 1974), a finding which resonates with the effectiveness of reappraisal in the regulation of other types of emotion (e.g., Jackson, Malmstadt, Larson, \& Davidson, 2000; Gross, 1998).

Reappraisal strategies can broadly be subdivided into (i) those that reinterpret negative aspects of stimuli as neutral or positive, such as when a woman's tears in front of a church are taken to signify joy in relation to somebody's wedding instead of sadness in relation to somebody's death (e.g., Ochsner, Bunge, Gross, \& Gabrieli, 2002); and (ii) those that accept the stimulus (and a potentially ensuing emotional reaction) as it is but deny its personal relevance, by taking a detached observer perspective (Eifert \& Heffner, 2003; Levesque et al., 2003; Beauregard, Levesque, \& Bourgouin, 2001). Reappraisal by reinterpretation has been called situation-focused reappraisal or redefinition; reappraisal by denial of relevance is also known as detachment, selffocused reappraisal, disengagement, dissociation, or isolation.

The neural systems subserving emotion regulation through reappraisal have been investigated during view- 
ing of emotionally negative pictures (Ochsner et al., 2002; Schaefer, Jackson, et al., 2002), and sexually arousing (Beauregard et al., 2001) or sad (Levesque et al., 2003) film excerpts. Regulation of anxiety has not been studied with neuroimaging so far. We chose an anticipatory anxiety for pain paradigm because pain is intrinsically aversive or, in other words, automatically appraised as personally (self) relevant. The paradigm is thus ideal for investigating the effects of reappraisal by denial of relevance (detachment). This is not the case where emotions are elicited by viewing of pictures of films where the personal relevance of the emotional stimuli is at best unclear.

The above studies have observed a pattern of prefrontal activations, and subcortical deactivations, leading to a suggestion that the former reflect a regulatory source and the latter regulatory sites. On the basis of previous fMRI studies on pain anticipation (Wager et al., 2004; Jensen et al., 2003; Porro et al., 2002; Phelps et al., 2001; Ploghaus, Tracey, et al., 1999), we conjectured that two brain regions-the medial prefrontal/anterior cingulate cortex (MPFC/ACC) and the right anterior insula-play a central role in anticipatory anxiety. We thus hypothesized that successful anxiety regulation would be associated with reduced activation in these two regions. We further predicted that prefrontal regions would show enhanced activity during regulation of anxiety.

In our experiment, subjects were forewarned they might receive an electric pain stimulus to the hand at a probability of $25 \%$ at any time during a 15.6 -sec epoch (Anxiety condition). During a control condition (Noanxiety), subjects were told they would not be stimulated. In a fully balanced, $2 \times 2$ factorial design, subjects had either to emotionally detach from their anxiety (Regulation condition) or to actively focus on the engendered emotion (No-regulation). In such a design, reduction of anticipatory anxiety due to the regulatory strategy should be evident from the interaction term.

\section{RESULTS}

\section{Behavioral Experiment}

Figure 1A shows the experimental design. Subjects $(n=18)$ rated their state anxiety at the beginning of the experiment as $30.2 \pm 1.6$ (SEM) and their trait anxiety as $34.9 \pm 1.8$. Thus, they deviated less than one standard deviation from a normal working adult population (Spielberger, 1983).

Subjective anxiety ratings of the four experimental conditions (Figure 2A) showed significant main effects of anxiety $[F(1,17)=103.2, p<.001]$ and regulation $[F(1,17)=34.8, p<.001]$ and a significant interaction $[F(1,17)=33.2, p<.001]$, driven by an attenuation of anxiety in the regulation condition. Blockwise averaged heart rate levels (HRLs; Figure 2B) were decreased by regulation [main effect: $F(1,17)=48.8, p=.036$ ]. Skin conductance levels (SCLs; Figure 2C) showed a significant main effect of anxiety $[F(1,17)=6.6, p=.02]$ and a significant interaction $[F(1,17)=5.2, p=.036]$, with a pattern similar to subjective ratings.

It has been reported that physiological responses to aversive events are less pronounced in a low compared to a high anxiety condition (Epstein \& Clarke, 1970). In line with these observations, and supporting an anxiolytic effect of regulation, there was a strong trend for attenuation of the phasic accelerative heart rate response (HRR) to actual receipt of pain during Anxiety/ Regulation relative to Anxiety/No-regulation (Figure 3A; $p=.054, t$ test, one-tailed, $n=6$ pain stimuli per condition and subject). Skin conductance responses (SCR) did not show a similar pattern of attenuation $(p=.132$; Figure 3B). Taken together, our behavioral data indicate that anticipation of pain induced an anxiety
Figure 1. Design and analysis. (A) The study involves a $2 \times 2$ factorial design, with factors Anxiety (No-anxiety vs. Anxiety) and Regulation (No-regulation vs. Regulation). Anxiety was induced by forewarning subjects with a high-pitch double-beep that they might receive an electric pain stimulus during the following $15.6 \mathrm{sec}$. Safe (No-anxiety) blocks were announced by a low-pitch double-beep. The regulation factor was operationalized in "mega-blocks" which spanned 8 blocks of Anxiety/No-anxiety. During regulation (announced by "Special Place") subjects had to detach from anxiety; during no-regulation ("Queen Square") subjects had to identify with their emotional state (see Methods). (B) Neural activations during blocks of Anxiety/No-anxiety were modeled as tonic, phasic, and linearly increasing and decreasing responses (see Methods).

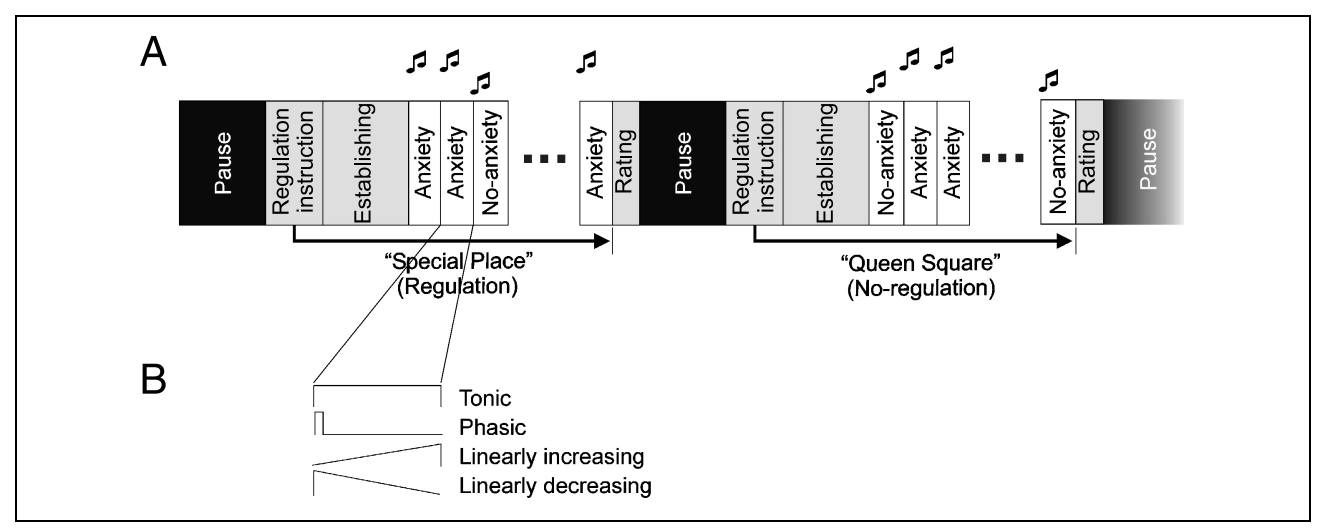




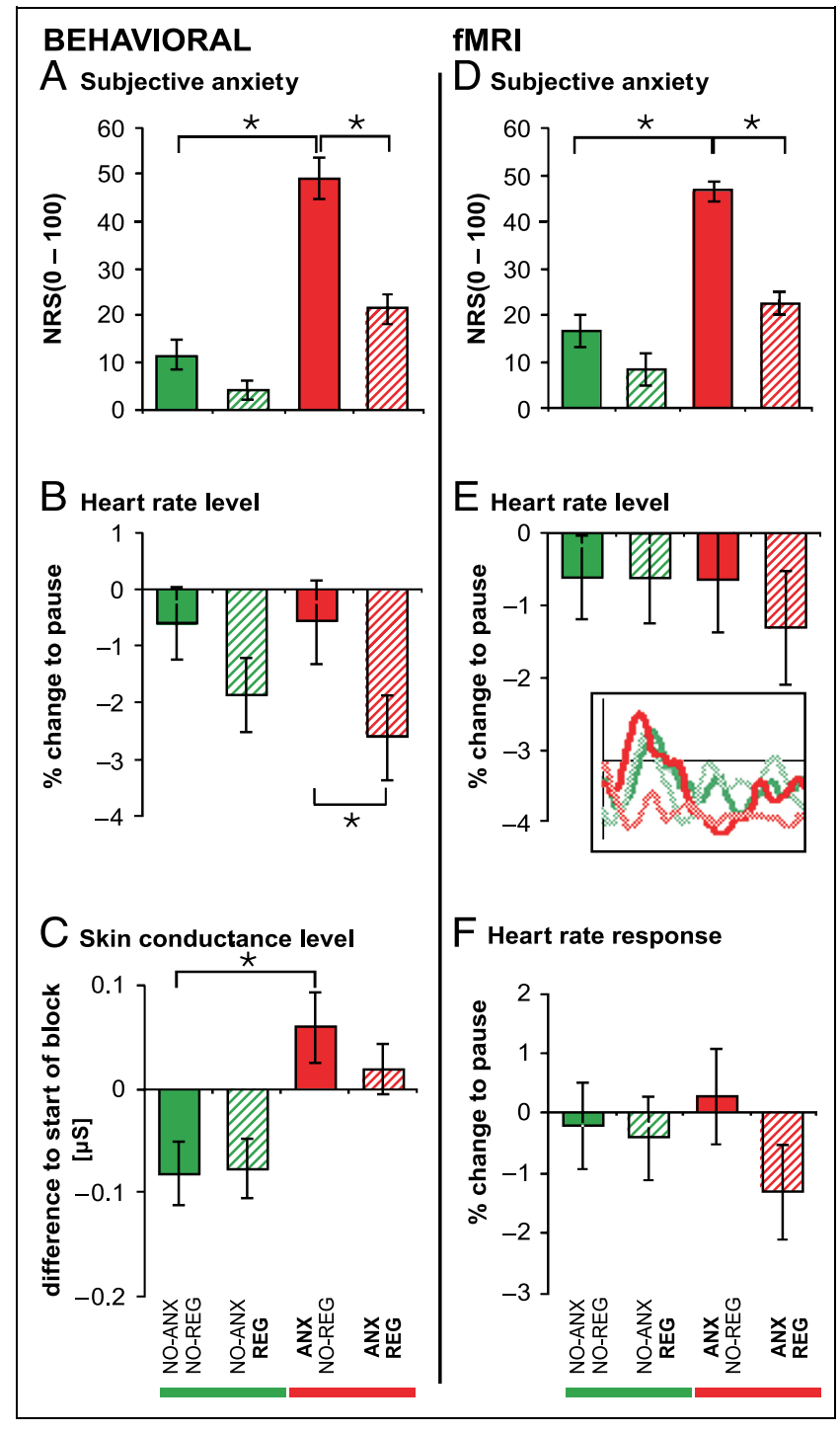

Figure 2. Physiological responses to anticipation of pain. Reduction of anxiety responses through regulation was evident both in the behavioral (A-C) and the fMRI experiment (D-F). Insert in (E) shows average HR time courses during the four experimental conditions. HRRs in (F) reflect the initial phasic response. Solid green: No-anxiety/ No-regulation (NO-ANX/NO-REG); hatched green: No-anxiety/ Regulation (NO-ANX/REG); solid red: Anxiety/No-regulation (ANX/ NO-REG); hatched red: Anxiety/Regulation (ANX/REG). NRS(1-100): 100-point numerical rating scale. Values: mean \pm SEM. $* p<.05$.

response and this response was modulated by a regulation strategy.

\section{fMRI Experiment}

\section{Self-report}

Sixteen of the subjects tested behaviorally participated in the fMRI experiment. Their state and trait anxiety scores were comparable to those reported for our behavioral experiment $(29.4 \pm 1.6$ and $33.4 \pm 1.6$, respectively). Similarly, subjective anxiety ratings of the four experimental conditions resembled the behavioral experiment with main effects of anxiety, regulation, and a significant interaction [Figure 2D; main effect of anxiety: $F(1,15)=92.0, p<.001$; of regulation: $F(1,15)=40.2, p<.001$; interaction: $F(1,15)=22.5$, $p=.001]$. All subjects who achieved reduction of subjective anxiety in the behavioral experiment also achieved anxiety reduction during imaging.

\section{Physiological Responses}

As in the behavioral experiment, HRL was not changed by anxiety; however, regulation failed to produce the above HRL decreases (Figure 2E). Unlike in the behav-

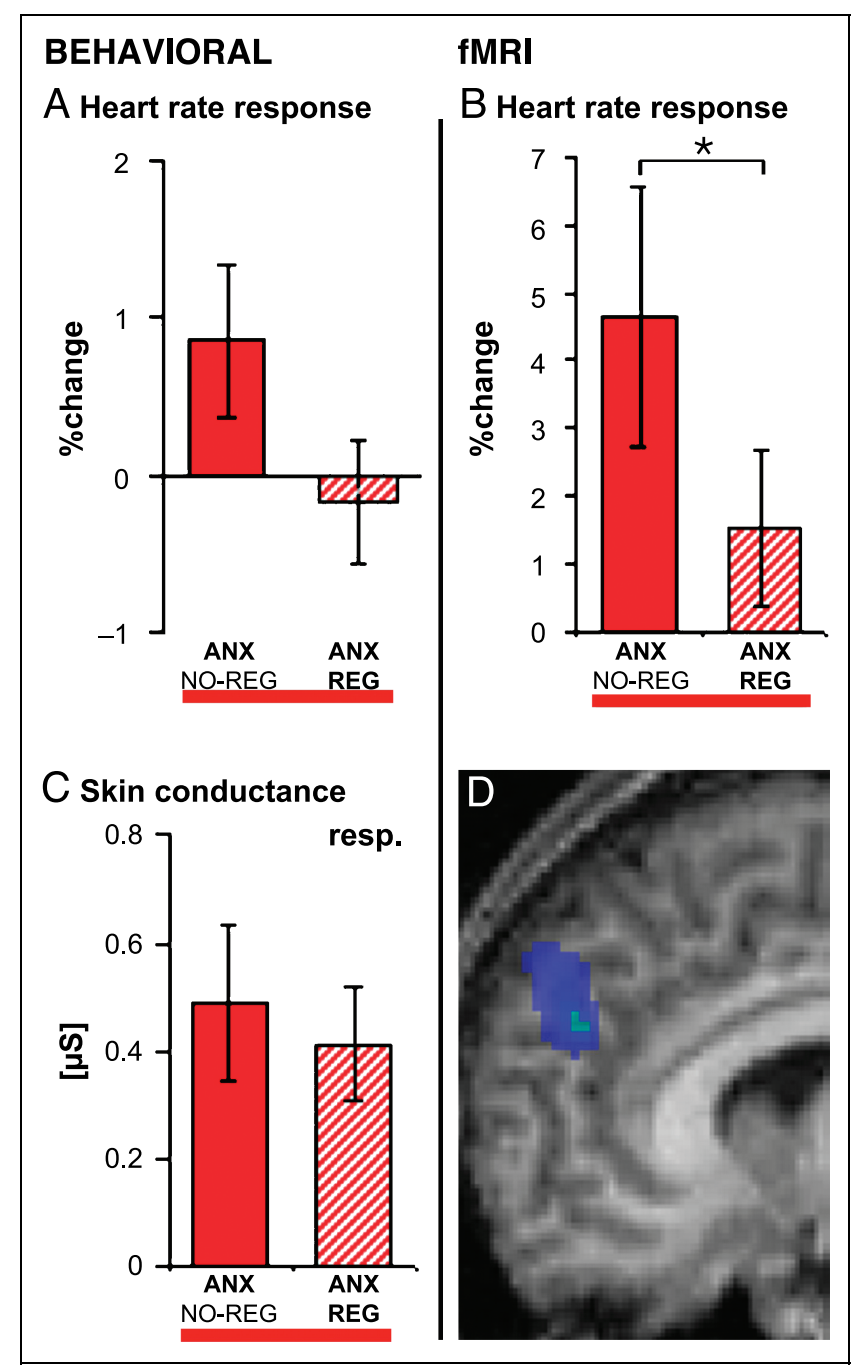

Figure 3. Physiological and neural responses to pain. Physiological (A-C) and neural (D) reactivity to pain was attenuated during Anxiety/ Regulation (hatched red, ANX/REG) relative to Anxiety/No-regulation (solid red, ANX/NO-REG), further supporting success of anxiety reduction through regulation. (A-C) Values: mean \pm SEM. $* p<.05$. (D) Right MPFC: maximum at $(6 / 52 / 28), z=5.14$; blue: $p<.001$, green: $p<.05$ (whole brain corrected). Activation superimposed on one subject's normalized structural image. 
ioral experiment, time courses showed an initial phasic heart rate acceleration (Figure $2 \mathrm{E}$ ). When restricting the analysis to the first 6 sec of a block, the response pattern resembled subjective ratings and a significant interaction between anxiety and regulation was observed $[F(1,13)=$ $5.1, p=.041$; Figure $2 \mathrm{~F}]$. As in the behavioral study, the phasic pain-induced accelerative HRRs were attenuated by regulation $(p=.047$; Figure $3 \mathrm{~B})$.

\section{Neuroimaging-Pain}

The attenuated physiological reactivity to pain during regulation relative to no-regulation predicts attenuated neural responses in pain regions. When receipt of pain was modeled as distinct events, the contrast

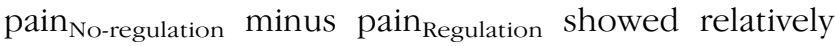
decreased signal for the latter condition in somatomotor and medial prefrontal regions. Attenuation was particularly pronounced in the MPFC (Figure 3D), a region previously implicated in affective aspects of pain processing (Rainville, Duncan, Price, Carrier, \& Bushnell, 1997). This is consistent with the idea that background mood state modulates the affective rather than sensory-discriminative aspects of pain perception (Villemure \& Bushnell, 2002).

\section{Neuroimaging-Anticipation of Pain}

Given the lengthy blocks of anticipation (16 sec) and under an assumption that the neural responses might not be constant across time, we modeled activity during each of the four experimental conditions as three separate regressors: tonic, phasic, and linear (increasing or decreasing) (Figure 1 and Methods). To identify regulation-dependent reductions of anxiety responses, we tested for the interaction: (Anxiety $>$ No-anxiety $)_{\text {No-regulation }}>(\text { Anxiety }>\text { No-anxiety })_{\text {Regulation }}$. A priori regions of interest (ROIs) were defined in the left and right MPFC/ACC and in the right anterior insula (see Methods). A cluster exhibiting three maxima was found for a phasic response in the MPFC/ACC ROI which also survived small volume correction at $p=.05$. Two of the peaks fulfilled the additional criterion of showing simple main effects of both anxiety (Anxiety/No-regulation $>$ No-anxiety/No-regulation) and regulation under anxiety (Anxiety/Regulation < Anxiety/No-regulation) in post hoc $t$ tests on parameter estimates (Figure 4). The parameter estimates as shown in Figure $4 \mathrm{~B}$ are consistent with a reduction of anxietyinduced activation as a consequence of regulation. MPFC/ACC activation as a consequence of anxiety (in the contrast Anxiety/No-regulation > No-anxiety/Noregulation) showed laterality effects. Parameter estimates of the side contralateral to the pain-receiving hand were significantly higher than on the ipsilateral side $(p=.042$, two-tailed, at voxel $\pm 4 / 46 / 28)$.
The interaction (Regulation $>$ No-regulation) Anxiety $>$

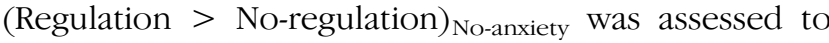
identify regions engaged specifically during regulation of anxiety. Within the prefrontal cortex, interactions were found for a tonic response in the left orbitofrontal cortex (OFC; Figure 5C) and a linearly increasing response in the right anterolateral PFC (Figure 5A). Activation patterns are consistent with a role of the anterolateral PFC (Figure 5B), but not the OFC (Figure 5C), as a higher-order cognitive region specifically activated during an attempt to regulate anxiety.

\section{DISCUSSION}

Reappraisal by denial of relevance ("taking a detached observer position") implicitly or explicitly involves generating an image of the (observing) self which is unaffected by (the observed) external circumstances. The strategy as operationalized here (see Methods for details) relied upon explicit generation of an experiencing self detached from the immediate surroundings and any disturbing emotions (the "special place" condition). It was compared to a condition ("Queen Square") in which subjects remain engaged with their surroundings and with their emotional reactions, which they related to their own self. In both conditions, the associated self statements encouraged subjects to be aware of emotional reactions. We expected the explicit generation of a detached self to facilitate reappraisal (i.e., denial of the personal relevance of pain warnings and emotional reactions), and this to manifest in reductions in subjective experience of anxiety, sympathetic arousal, pain reactivity, and anxiety-related neural activity. Obviously, denial of personal relevance of a stimulus (or reaction) can only be meaningful if the stimulus carries personally relevant information. This is assured in our experiment where the stimuli carry a prediction of pain.

Subjective reports of anxiety regulation effects were validated by additionally recording physiological measures and neural reactivity to pain. Because of interindividual variability in the use of self-report scales and the possibility of subjects giving desirable answers, the critical evaluation of self-report is of particular importance. Physiologically, the unregulated anxiety condition (Anxiety/No-regulation) was characterized by (i) unchanged HRLs; (ii) initial phasic heart rate accelerations (observed during the fMRI experiment only); and (iii) increases in SCLs (measured in the behavioral experiment only). Ploghaus, Narain, et al. (2001) have reported both HRL decreases (during a low anxiety condition) as well as HRL increases (during a high anxiety condition) in anticipation of pain. The absence of HRL changes (see i) in our study is therefore consistent with no anxiety or with (the intended) intermediate level of anxiety. However, in combination with the subjective ratings, the 


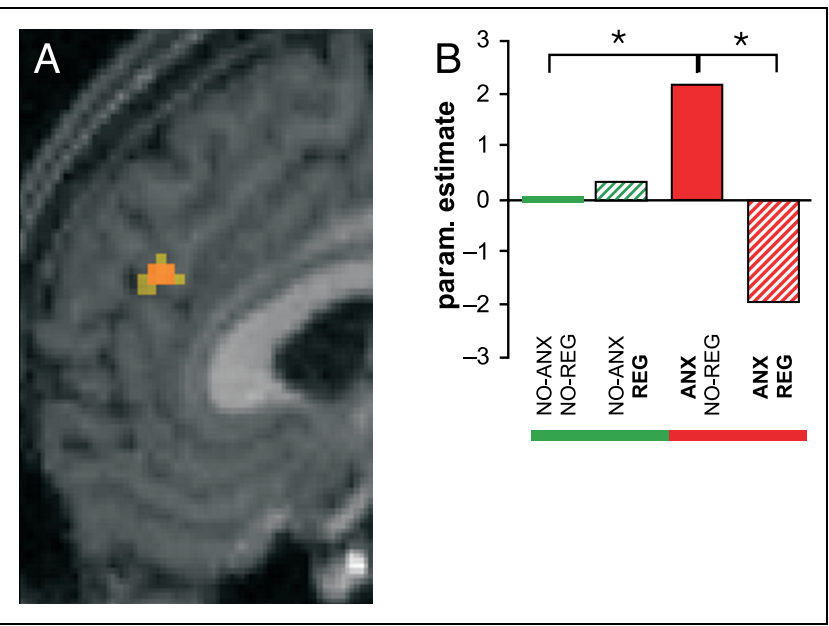

Figure 4. Regulatory site: Interaction in the MPFC/ACC. (A) Maximum shown is at $(-4 / 46 / 28), z=3.83$; yellow: $p<.001$, red: $p<.05$ (small volume corrected). (B) Mean parameter estimates relative to No-anxiety/No-regulation show reduction of anxiety-evoked MPFC/ACC activation as a consequence of regulation. ${ }^{*} p<.05$ ( $t$ test, one-tailed).

initial heart rate accelerations (ii) and the sustained SCL increases (iii), which are suggestive of a tonic arousal state (Barry, 1996), we can safely conclude anxiety has been induced.

The appearance of initial phasic heart rate accelerations to the warning signals (ii) in the fMRI experiment suggests these cues were perceived as more salient than in the behavioral study. This may be due to elevated anxiety levels in the scanner (Friday \& Kubal, 1990) and may also explain the failure of regulation to induce HRL decreases in the fMRI study. In support of this interpretation, subjective ratings of the baseline condition (Noanxiety/No-regulation) showed a trend to be higher in the fMRI experiment ( $p=.069$, paired $t$ test, onetailed). An alternative explanation may be that subjects had to be more vigilant in the scanner to detect the auditory cues against a background of scanner noise.

Regulation reduced (i) the phasic heart rate accelerations to the pain warnings, (ii) SCLs during anxiety, and (iii) HRRs and cerebral activation to pain. Reduction of SCRs to pain did not reach significance. Thus, there is strong evidence for regulation-dependent reductions in anxiety, in concordance with subjective ratings.

The use of a full $2 \times 2$ factorial design allowed us to differentiate general effects of regulation, that is, the generation and maintenance in working memory of a state of detachment (in the main effect of regulation), from specific effects of regulation during anxiety [i.e., the reappraisal as irrelevant of pain warnings and potential emotional reactions on the basis of a detached perspective (in the interaction term)]. Previous emotion regulation studies have only compared an unregulated with a regulated emotional state which does not allow such differentiation. The right anterolateral PFC region, identified as a "regulatory site," has also been found by Beauregard et al. (2001) during reappraisal of erotic stimuli and Schaefer, Collette, et al. (2003) during propositional versus schematic emotional processing. A slightly more dorsocaudal region was found by Levesque et al. (2003) during reappraisal of sadness-inducing stimuli. Ochsner et al. (2002) described a similar, albeit left-sided, region during reappraisal of emotionally negative pictures. In addition, some of these studies reported other lateral PFC regions as well as bilateral caudal dorsal MPFC (Ochsner et al., 2002), right OFC (Levesque et al., 2003), and rostral ACC (Beauregard et al., 2001) during the above conditions. This suggests that anterolateral PFC recruitment is a common denominator of emotion regulation by reappraisal, independent of strategy and emotional stimulus. The left-sided activation in Ochsner et al. may reflect use of a reappraisal by reinterpretation strategy which consists in finding an alternative narrative for affective picture

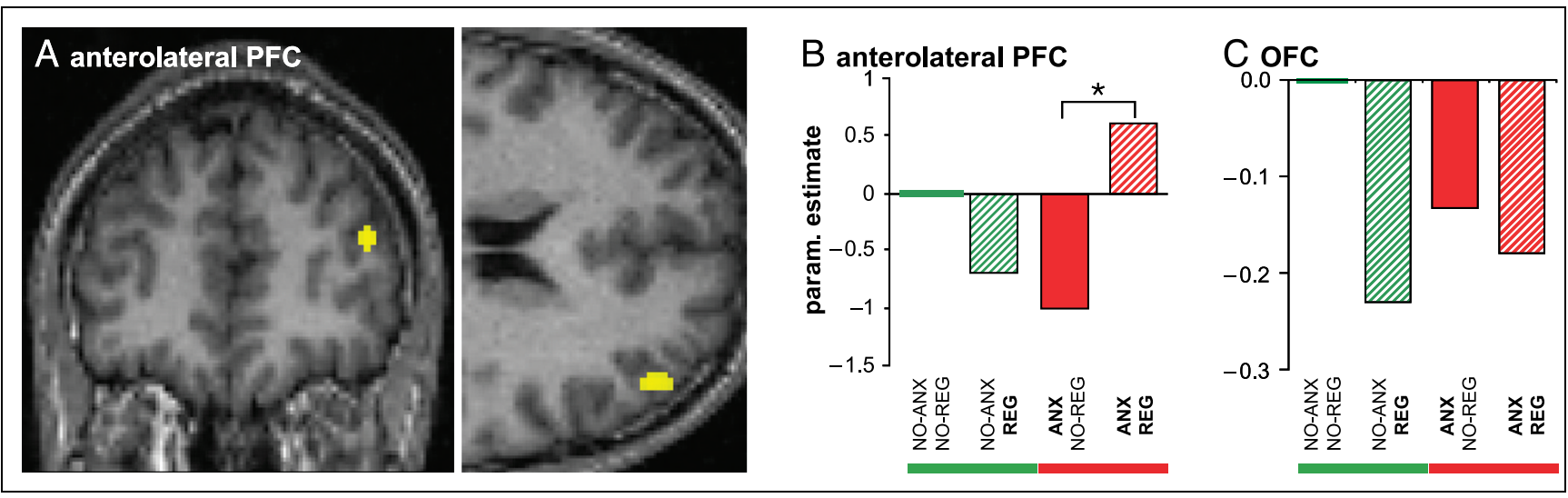

Figure 5. Regulatory source: Interaction in the anterolateral PFC. (A) Right anterolateral PFC (42/48/18), $z=3.62$; yellow: $p<.001$. (B) Mean parameter estimates relative to No-anxiety/No-regulation show activation of the anterolateral PFC during regulation of anxiety. ${ }^{*} p<.05(t$ test, one-tailed). (C) For comparison, parameter estimates in the left orbitofrontal cortex $(-32 / 46 /-2)$, inconsistent with a role in anxiety regulation, are shown. 
content and thus activates left-lateralized verbal/semantic processes (Gabrieli, Poldrack, \& Desmond, 1998; Tulving, Kapur, Craik, Moscovitch, \& Houle, 1994). By contrast, the reappraisal by denial of relevance strategy used in the present study depended upon, in addition to rehearsal of a verbal self-statement, recall of a pleasant interoceptive (relaxed body state) and exteroceptive (visual, auditory, spatial) experience (the "special place"). The strategy may therefore more strongly rely on right-lateralized episodic memory retrieval (Tulving et al., 1994) and processing of nonverbal material (Smith \& Jonides, 1999; D'Esposito et al., 1998). The increase in anterolateral PFC activity during the blocks may reflect the increasing demands of regulation across time in the face of a potential aversive stimulus.

Anxiety reduction as well as decreased pain reactivity are both reflected in attenuation of phasic MPFC/ACC activation. The MPFC/ACC is the brain region most consistently activated across many types of emotion induction paradigms (Murphy, Nimmo-Smith, \& Lawrence, 2003; Phan, Wager, Taylor, \& Liberzon, 2002). Specifically, the rostral area found here falls into the "affective" division of the ACC (Vogt, 1993), which is activated by emotional and deactivated by cognitive tasks (Bush, Luu, \& Posner, 2000). Its putative functions include evaluation of emotional-motivational relevance of stimuli (Devinsky, Morrell, \& Vogt, 1995), which is consistent with its activation during processing of selfreferential stimuli (Kelley et al., 2002), generation of arousal (Critchley, 2004), allocation of attentional resources to salient stimuli (Niedenthal \& Kitayama, 1994), internal generation of emotions (Phan, Wager, et al., 2002), affective experience (Rainville et al., 1997), and attention to affective experience (Taylor, Phan, Decker, \& Liberzon, 2003; Lane, Fink, Chau, \& Dolan, 1997).

The transient activation of the MPFC/ACC, reflected in the phasic regressor, suggests a function early in the anticipatory process such as an evaluation of the motivational/self relevance of the auditory cues, orienting of attention towards the locus of expected pain, or avoidance response preparation. Alternatively, the MPFC/ACC may simply generate the observed phasic HRRs (Critchley, 2004). Importantly, activation of the MPFC/ACC by emotional stimuli is dependent on context factors which modulate their salience. It is increased by attention (Fichtenholtz et al., 2004; Taylor et al., 2003; Lane et al., 1997) and decreased by repeated exposure to emotional material (Phan, Liberzon, Welsh, Britton, \& Taylor, 2003). We suggest that denial of relevance of pain warnings, facilitated by a detached mental state, decreases their salience and this is reflected in attenuated MPFC/ACC activation.

In conclusion, we show that a regulatory strategy of detachment attenuates subjective and physiological indices of anxiety. Under similar experimental conditions, we have identified a putative neural source and site of anxiety of this regulation. Ultimately, this line of re- search can lay the ground for investigating emotion regulation deficits in patients and for empirical validation of putative therapeutic regulation strategies.

\section{METHODS}

\section{Subjects}

Eighteen right-handed healthy normal subjects (mean age 27 years, age range 20-34 years, 10 women) participated in the experiment. The subjects were preassessed to exclude those with a prior history of neurological or psychiatric illness, including anxiety disorders. All subjects gave informed consent, and the study was approved by the Joint Ethics Committee of the National Hospital for Neurology and Neurosurgery.

\section{Anxiety Reduction Strategy}

In a group session prior to behavioral testing and prior to the fMRI experiment, subjects were taught a strategy of detachment based on an established clinical anxiety management technique (Walters \& Oakley, 2003; Oakley, 1998). They were asked to identify a safe and relaxing place of their own choosing (their "Special Place") and to bring this place to mind as vividly as possible in all sensory modalities. They were also asked to create a self-statement describing the experience of safety and relaxation based on the generic form: "I am here in my ['Special Place'], feeling safe and comfortable. Those emotions out there cannot reach me here. Nothing bothers me." They were then guided through a stepwise mental and muscular relaxation procedure leading them into their "Special Place" and allowing them to mentally explore it in a state of focused attention and heightened absorption, again using all sensory modalities, and to associate that particular mental state with the "Special Place" self-statement. They were encouraged to visualize unwanted feelings or thoughts as objects in a far distance from their special place (e.g., as a cloud far out on the horizon while lying on a beautiful beach). Finally, subjects were asked to occasionally "go into" their special place and rehearse the related self-statement in their daily life and to try and use this strategy in order to detach from negative feelings. They were told the fMRI experiment would only be performed in case of success in prior behavioral testing.

During the experiment, subjects were instructed to return to the "Special Place" experience and to subvocally repeat the self-statement when cued to do so by hearing the words "Special Place." In the control condition, subjects were instructed to imagine themselves in their actual surroundings at the laboratory at Queen Square and use a corresponding self-statement: "I am now in this experiment at Queen Square. I can clearly 
feel my emotions. They affect my body and my mind" when cued to do so by hearing the words "Queen Square." Both conditions were intended to be similar in terms of subvocal rehearsal, visual imagery, emotional awareness, and acceptance of emotional stimuli and reactions (see Discussion).

\section{Experimental Design}

The experimental design was identical for the behavioral and fMRI experiments (Figure 1A). Pain stimuli were applied to the back of the left or right hand (balanced between subjects) using a custom-built electrical stimulator delivering 20 or $100 \mathrm{~Hz}$ trains of electrical pulses (4 msec monopolar square waveform pulses, 1 sec duration) through a silver chloride electrode. Current levels (ranging between 0.1 and $6 \mathrm{~mA}$ ) and stimulation frequencies were chosen which induced intermediate subjective anxiety. To achieve this, subjects rated their anxiety during a 16-to-0 countdown on a 100-point scale. Subjects were told they might receive a previously experienced painful stimulus at any time during the countdown at a probability of $25 \%$. This procedure was repeated with different current levels, starting at low levels, until an anxiety level between 30 and 60 was reached.

During the actual experiment, subjects were lying on their back with eyes closed and headphones on. The experiment was split into three runs of approximately 10,13 , and 10 min duration. Runs consisted of 3, 4, and 3 mega-blocks of 3 min each during which subjects either had to employ the detachment strategy (Regulation condition, 5 mega-blocks) or the control strategy (No-regulation condition, 5 mega-blocks). The sequence of these conditions was randomized.

Each mega-block started with a pause of $31 \mathrm{sec}$. Subjects then received the instruction "Special Place" (Regulation condition) or "Queen Square" (No-regulation condition) over the headphones and were given $30 \mathrm{sec}$ to fully establish the associated mental state. This was followed by 8 pseudorandomized blocks of $15.6 \mathrm{sec}$ each during which subjects knew they might receive a pain stimulus (Anxiety condition, 25\% probability of stimulus at any time) or not (No-anxiety condition). Anxiety blocks were announced by a high-pitch double beep, No-anxiety blocks by a low-pitch double beep. Thus, there were four experimental conditions (Noanxiety/No-regulation, $n=17$; No-anxiety/Regulation, $n=17$; Anxiety/No-regulation, $n=23,6$ pain stimuli; Anxiety/Regulation, $n=23,6$ painful stimuli). At the end of a mega-block, subjects were given $6 \mathrm{sec}$ to indicate how much of the time after the instruction they used the corresponding strategy and self-statement $(0=$ not at all, $1=$ roughly one third of the time, $2=$ roughly two thirds of the time, $3=$ the whole time; headphone instruction: "rating"). This rating was given verbally during the behavioral experiment and via a button response box during the fMRI experiment. It served to verify that subjects had not fallen asleep and were paying attention to the instructions. After each run subjects rated their anxiety during the four conditions on the 100-point scale. They were reminded not to rate their affective responses to the actual pain. Current levels were adjusted between experimental runs if subjective ratings in the Anxiety minus No-anxiety comparison during the No-regulation condition differed markedly from the previously calibrated value.

\section{Behavioral Experiment}

Behavioral testing was performed 1 week after the group training session in individual sessions. Prior to testing, subjects completed Spielberger's trait and state anxiety inventories (STAI-S, STAI-T, Mind Garden, Redwood, CA, USA). Subjects were then explained the setup for autonomic monitoring and told autonomic measures were the only objective way to verify success of anxiety reduction. Subjects again underwent the relaxation procedure leading them into their special place in order to refresh their memory of the mental state associated with the detachment condition and were then reoriented to the experimental setting. After current level calibration, they performed one practice run of about $10 \mathrm{~min}$ without pain stimulation prior to the actual experiment. Heart rate (HR) was monitored using a pulse oximeter (Nonin 8600FO, Nonin Medical, Plymouth, MN, USA); the pulse probe was placed on the index finger of the hand opposite to the stimulated hand. Galvanic SCLs were recorded using an AT64 SCR apparatus (Autogenic Systems, Wood Dale, IL, USA); electrodes were placed on the middle and ring fingers of the unstimulated hand.

\section{fMRI Experiment}

fMRI was performed between 3 and 25 days after behavioral testing. One subject was unavailable, another subject was excluded due to failure of subjective anxiety reduction. The remaining 16 subjects (mean age 26 years, age range 20-35 years, 9 women) completed the STAI questionnaires and a social desirability questionnaire (results will be presented elsewhere). They were again led through the relaxation and special place procedure as a reminder of the detachment condition and were then reoriented. This was followed by current level calibration and a practice run within the scanner. HR was monitored using an identical setup. A 1.5-T Siemens Sonata MRI scanner was used to acquire gradient-echo $\mathrm{T} 2 *$-weighted echo-planar images (EPI) images with BOLD (blood oxygenation level-dependent) contrast $(\mathrm{TE}=50 \mathrm{msec}, \mathrm{TR}=90 \mathrm{msec}$, flip angle $=$ $90^{\circ}$ ). Each volume comprised 44 tilted slices of $2 \mathrm{~mm}$ thickness and $3 \times 3 \mathrm{~mm}^{2}$ in-plane resolution with a slice distance of $1 \mathrm{~mm}$. A total of 514 volumes, distributed 
over three runs (155, 204, 155 volumes), were acquired continuously every $3.96 \mathrm{sec}$. These parameters produced EPI images in which signal dropout from susceptibility artifact was restricted to far caudal OFC, leaving the remaining sectors of OFC intact (Deichmann, Gottfried, Hutton, \& Turner, 2003). Subjects were placed in a light head restraint within the scanner to limit head movement during acquisition. A T1-weighted structural image was also acquired (Deichmann, Schwarzbauer, \& Turner, 2004).

\section{Data Analysis}

The subjective anxiety ratings obtained at the end of each run were weighted by the number of regulation or no-regulation mega-blocks within that run before averaging across the whole experiment. Raw HR waveforms were visually inspected and excluded where automatic pulse detection was inaccurate (2 subjects in the fMRI experiment). Blockwise averaged HRLs were normalized to the last $20 \mathrm{sec}$ of the pause at the beginning of each mega-block; initial phasic HRRs were taken from 1 to $6 \mathrm{sec}$ after start of block and normalized identically. Blockwise SCLs were normalized to the first value in a block in order to exclude drift-related confounds. Blocks where subjects actually received pain stimuli were excluded from the analysis. HRRs and SCRs to pain stimulation were calculated as average HR or skin conductance during the poststimulus window which showed maximum physiological activation in the group data, normalized to the second prior to stimulus. The time window was of $1 \mathrm{sec}$ duration for HRR and $3 \mathrm{sec}$ duration for SCR to account for the slower dynamics of skin conductance changes. Statistical inference was based on one-way analysis of variance (ANOVA) with repeated measures and Student's $t$ test within SPSS 11.

Imaging data were analyzed using SPM2 (www.fil.ion. ucl.ac.uk/spm/spm2; Frackowiak, Friston, Frith, et al., 2004). The four initial images of each run were discarded. Images were realigned to the fifth volume of the first run, spatially normalized to a standard T2* template, spatially smoothed using a Gaussian kernel with a full width at half maximum (FWHM) of $6 \mathrm{~mm}$, temporally high-pass filtered (cutoff $128 \mathrm{sec}$ ) and corrected for temporal autocorrelations. Statistical analysis was carried out by applying a random effects analysis using the general linear model across the 16 subjects. Each of the four experimental conditions was modeled using three different temporal profiles of neuronal response during the 15.6-sec block: a phasic response occurring at the beginning of the block, a tonic neuronal response lasting the whole duration of the block, and a linearly increasing response across the block (Figure 1B). Multiplication of the linearly increasing regressor by -1 in the definition of contrasts (see below) allowed assessment of linearly decreasing effects.
Receipt of pain and instructions were modeled as distinct events. Blocks during which subjects actually received pain stimuli; times for establishing the mental state at the beginning of the mega-blocks; and ratings were modeled as box-car regressors. To retain degrees of freedom, the regressors for the three runs were concatenated. Residual motion effects were corrected for by including the six estimated motion parameters for each subject as regressors in the model. Each regressor was convolved with the canonical hemodynamic response function. Calculation of voxelwise within-subject effects of linear combinations of the regressors yielded contrast images that were spatially smoothed (FWHM $10 \mathrm{~mm}$ ), resulting in an estimated smoothness of 10 $11 \mathrm{~mm}$, and compared between subjects using onesample $t$ tests.

Clusters with $>5$ voxels activated at a statistical threshold of $p=.001$ are reported. Correction for multiple comparisons following Gaussian random field theory was limited to two spherical regions of interest in which interactions of the type (Anxiety $>$ Noanxiety) $)_{\text {No-regulation }}>$ (Anxiety $>$ No-anxiety) $)_{\text {Regulation }}$ were expected: MPFC/ACC $( \pm 6 / 44 / 32)$ and right anterior insula (36/17/6). A relatively large diameter $(24 \mathrm{~mm})$ was chosen to account for interindividual anatomical variability in frontal regions. Coordinates were averages derived from three previous anticipatory anxiety studies (Jensen et al., 2003; Porro et al., 2002; Ploghaus, Tracey, et al., 1999). The other anticipatory anxiety studies in the literature (Wager et al., 2004; Phelps et al., 2001) do not report coordinates and were not considered. Talairach coordinates were transformed into MNI (Montreal Neurological Institute) space using a standard algorithm (eeg.sourceforge.net/mridoc/mri_toolbox/ tal2mni.html). Because in two studies (Jensen et al., 2003; Ploghaus, Tracey, et al., 1999) only the left hand was stimulated, we also tested the ROIs contralateral to the reported coordinates.

Anatomical localization was carried out with reference to the atlas of Duvernoy (1999). To illustrate group effect sizes in selected voxels, mean parameter estimates from the main effect of a second-level oneway analysis of variance over the four experimental conditions were used. These parameter estimates were also used for post hoc $t$ tests.

\section{Acknowledgments}

The authors thank V. Walters and M. Whalley for help in developing the detachment strategy, P. Aston and E. Featherstone for experimental help, and $\mathrm{H}$. Ehrsson for help with anatomical localization. This work was supported by grants from the Marie Curie programme (RK), Deutsche Forschungsgemeinschaft $(\mathrm{KW})$, and the Wellcome Trust (BS, HC, JOD, RJD).

Reprint requests should be sent to Raffael Kalisch, Functional Imaging Laboratory, Wellcome Department of Imaging Neuro- 
science, University College London, London WC1N 3BG, UK, or via e-mail: rkalisch@fil.ion.ucl.ac.uk.

\section{REFERENCES}

Barry, R. J. (1996). Preliminary process theory: Towards an integrated account of the psychophysiology of cognitive processes. Acta Neurobiologiae Experimentalis (Wars.), 56, 469-484.

Beauregard, M., Levesque, J., \& Bourgouin, P. (2001). Neural correlates of conscious self-regulation of emotion. Journal of Neuroscience, 21, RC165.

Bush, G., Luu, P., \& Posner, M. I. (2000). Cognitive and emotional influences in anterior cingulate cortex. Trends in Cognitive Sciences, 4, 215-222.

Cannon, W. B. (1929). Bodily changes in pain, hunger, fear and rage. New York: Appleton.

Critchley, H. D. (2004). The human cortex responds to an interoceptive challenge. Proceedings of the National Academy of Sciences, U.S.A., 101, 6333-6334.

Deichmann, R., Gottfried, J. A., Hutton, C., \& Turner, R. (2003). Optimized EPI for fMRI studies of the orbitofrontal cortex. Neuroimage, 19, 430-441.

Deichmann, R., Schwarzbauer, C., \& Turner, R. (2004). Optimisation of the 3D MDEFT sequence for anatomical brain imaging: Technical implications of 1.5 and $3 \mathrm{~T}$. Neuroimage, 21, 757-767.

D'Esposito, M., Aguirre, G. K., Zarahn, E., Ballard, D., Shin, R. K., \& Lease, J. (1998). Functional MRI studies of spatial and nonspatial working memory. Brain Research, Cognitive Brain Research, 7, 1-13.

Devinsky, O., Morrell, M. J., \& Vogt, B. A. (1995). Contributions of anterior cingulate cortex to behaviour. Brain, 118, 279-306.

Duvernoy, H. M. (1999). The buman brain: Surface, blood supply, and three-dimensional sectional anatomy. Wien: Springer.

Eifert, G. H., \& Heffner, M. (2003). The effects of acceptance versus control contexts on avoidance of panic-related symptoms. Journal of Behavior Therapy and Experimental Psychiatry, 34, 293-312.

Epstein, S., \& Clarke, S. (1970). Heart rate and skin conductance during experimentally induced anxiety: Effects of anticipated intensity of noxious stimulation and experience. Journal of Experimental Psychology, 84, 105-112.

Eysenck, M. W. (1997). Anxiety and cognition (pp. 27-52). Hove, UK: Psychology Press.

Fichtenholtz, H. M., Dean, H. L., Dillon, D. G., Yamasaki, H., McCarthy, G., \& LaBar, K. S. (2004). Emotion-attention network interactions during a visual oddball task. Brain Research, Cognitive Brain Research, 20, 67-80.

Frackowiak, R. S. J., Friston, K. J., Frith, C. D., Dolan, R. J., Price, C. J., Zeki, S., Ashburner, J., \& Penny, W. (2004). Human Brain Mapping, 2nd ed. San Diego, CA: Elsevier.

Friday, P. J., \& Kubal, W. S. (1990). Magnetic resonance imaging: Improved patient tolerance utilizing medical hypnosis. American Journal of Clinical Hypnosis, $33,80-84$

Gabrieli, J. D., Poldrack, R. A., \& Desmond, J. E. (1998). The role of left prefrontal cortex in language and memory. Proceedings of the National Academy of Sciences, U.S.A., 95, 906-913.

Gray, J. A., \& McNaughton, N. (2000). The neuropsychology of anxiety (2nd ed.) Oxford: Oxford University Press.

Gross, J. J. (1998). Antecedent- and response-focused emotion regulation: Divergent consequences for experience, expression, and physiology. Journal of Personality and Social Psychology, 75, 224-237.

Gross, J. J. (1999). Emotion regulation: Past, present, future. Cognition and Emotion, 13, 551-573.

Gross, J. J. (2002). Emotion regulation: Affective, cognitive, and social consequences. Psychophysiology, 39, 281-291.

Hofer, M. A. (2002). Evolutionary concepts of anxiety. In D. J. Stein \& E. Hollander (Eds.), Textbook of anxiety disorders (pp. 57-69). Washington: American Psychiatric Publishing.

Holmes, D. S., \& Houston, B. K. (1974). Effectiveness of situation redefinition and affective isolation in coping with stress. Journal of Personality and Social Psychology, 29, 212-218.

Jackson, D. C., Malmstadt, J. R., Larson, C. L., \& Davidson, R. J. (2000). Suppression and enhancement of emotional responses to unpleasant pictures. Psychophysiology, 37, 515-522.

Jensen, J., McIntosh, A. R., Crawley, A. P., Mikulis, D. J., Remington, G., \& Kapur, S. (2003). Direct activation of the ventral striatum in anticipation of aversive stimuli. Neuron, 40, 1251-1257.

Kelley, W. M., Macrae, C. N., Wyland, C. L., Caglar, S., Inati, S., \& Heatherton, T. F. (2002). Finding the self? An event-related fMRI study. Journal of Cognitive Neuroscience, 14, 787-794.

Lane, R. D., Fink, G. R., Chau, P. M., \& Dolan, R. J. (1997). Neural activation during selective attention to subjective emotional responses. NeuroReport, 8, 3969-3972.

Levesque, J., Eugene, F., Joanette, Y., Paquette, V., Mensour, B., Beaudoin, G., Leroux, J. M., Bourgouin, P., \& Beauregard, M. (2003). Neural circuitry underlying voluntary suppression of sadness. Biological Psychiatry, 53, 502-510.

Murphy, F. C., Nimmo-Smith, I., \& Lawrence, A. D. (2003). Functional neuroanatomy of emotions: A meta-analysis. Cognitive, Affective and Behavioral Neuroscience, 3, 207-233.

Niedenthal, P. M., \& Kitayama, S. (1994). The beart's eye-Emotional influences in perception and attention. New York: Academic Press.

Oakley, D. A. (1998). Emptying the habit: A case of trichotillomania. Contemporary Hypnosis, 15, 109-117.

Ochsner, K. N., Bunge, S. A., Gross, J. J., \& Gabrieli, J. D. (2002). Rethinking feelings: An fMRI study of the cognitive regulation of emotion. Journal of Cognitive Neuroscience, 14, 1215-1229.

Phan, K. L., Liberzon, I., Welsh, R. C., Britton, J. C., \& Taylor, S. F. (2003). Habituation of rostral anterior cingulate cortex to repeated emotionally salient pictures. Neuropsychopharmacology, 28, 1344-1350.

Phan, K. L., Wager, T., Taylor, S. F., \& Liberzon, I. (2002). Functional neuroanatomy of emotion: A meta-analysis of emotion activation studies in PET and fMRI. Neuroimage, 16, 331-348.

Phelps, E. A., O'Connor, K. J., Gatenby, J. C., Gore, J. C., Grillon, C., \& Davis, M. (2001). Activation of the left amygdala to a cognitive representation of fear. Nature Neuroscience, 4, 437-441.

Ploghaus, A., Narain, C., Beckmann, C. F., Clare, S., Bantick, S., Wise, R., Matthews, P. M., Rawlins, J. N., \& Tracey, I. (2001). Exacerbation of pain by anxiety is associated with activity in a hippocampal network. Journal of Neuroscience, 21, 9896-9903.

Ploghaus, A., Tracey, I., Gati, J. S., Clare, S., Menon, R. S., Matthews, P. M., \& Rawlins, J. N. (1999). Dissociating pain from its anticipation in the human brain. Science, 284, 1979-1981. 
Porro, C. A., Baraldi, P., Pagnoni, G., Serafini, M., Facchin, P., Maieron, M., \& Nichelli, P. (2002). Does anticipation of pain affect cortical nociceptive systems? Journal of Neuroscience, 22, 3206-3214.

Rainville, P., Duncan, G. H., Price, D. D., Carrier, B., \& Bushnell, M. C. (1997). Pain affect encoded in human anterior cingulate but not somatosensory cortex. Science, 277, 968-971.

Schaefer, A., Collette, F., Philippot, P., van der, L. M., Laureys, S., Delfiore, G., Dugueldre, C., Maquet, P., Luxon, A., \& Salmon, E. (2003). Neural correlates of "hot" and "cold" emotional processing: A multilevel approach to the functional anatomy of emotion. Neuroimage, 18, 938-949.

Schaefer, S. M., Jackson, D. C., Davidson, R. J., Aguirre, G. K., Kimberg, D. Y., \& Thompson-Schill, S. L. (2002). Modulation of amygdalar activity by the conscious regulation of negative emotion. Journal of Cognitive Neuroscience, 14, 913-921.

Smith, E. E., \& Jonides, J. (1999). Storage and executive processes in the frontal lobes. Science, 283, 1657-1661.

Spielberger, C. D. (1983). State-trait anxiety inventory (Form Y). Redwood City, CA: Mind Garden.

Taylor, S. F., Phan, K. L., Decker, L. R., \& Liberzon, I. (2003).
Subjective rating of emotionally salient stimuli modulates neural activity. Neuroimage, 18, 650-659.

Tulving, E., Kapur, S., Craik, F. I., Moscovitch, M., \& Houle, S. (1994). Hemispheric encoding/retrieval asymmetry in episodic memory: Positron emission tomography findings. Proceedings of the National Academy of Sciences, U.S.A., 91, 2016-2020.

Villemure, C., \& Bushnell, M. C. (2002). Cognitive modulation of pain: How do attention and emotion influence pain processing? Pain, 95, 195-199.

Vogt, B. A. (1993). Structural organization of cingulate cortex: Areas, neurons, and somatodendritic receptors. In B. A. Vogt \& M. Gabriel (Eds.), Neurobiology of cingulate cortex and limbic thalamus: A comprebensive handbook (pp. 19-70). Boston: Birkhaeuser.

Wager, T. D., Rilling, J. K., Smith, E. E., Sokolik, A., Casey, K. L., Davidson, R. J., Kosslyn, S. M., Rose, R. M., \& Cohen, J. D. (2004). Placebo-induced changes in FMRI in the anticipation and experience of pain. Science, 303, $1162-1167$.

Walters, V. J., \& Oakley, D. A. (2003). Does hypnosis make in vitro, in vivo? Hypnosis as a possible virtual reality context in Cognitive Behavioural Therapy for an environmental phobia. Clinical Case Studies, 2, 295-305. 